\title{
Von den Abgrüinden des menschlichen Daseins
}

\author{
In Solothurn hat der Arzt und Autor Peter Hänni seinen vierten Kriminalroman \\ vorgestellt: «Boarding Time»*. Die SÄZ war an der Vernissage dabei und hat Hänni \\ vorgängig zum Gespräch getroffen.
}

\section{Daniel Lüthi}

Text und Bilder

\footnotetext{
* Eine Rezension mit den Angaben zum Buch findet sich im nächsten Artikel.
}

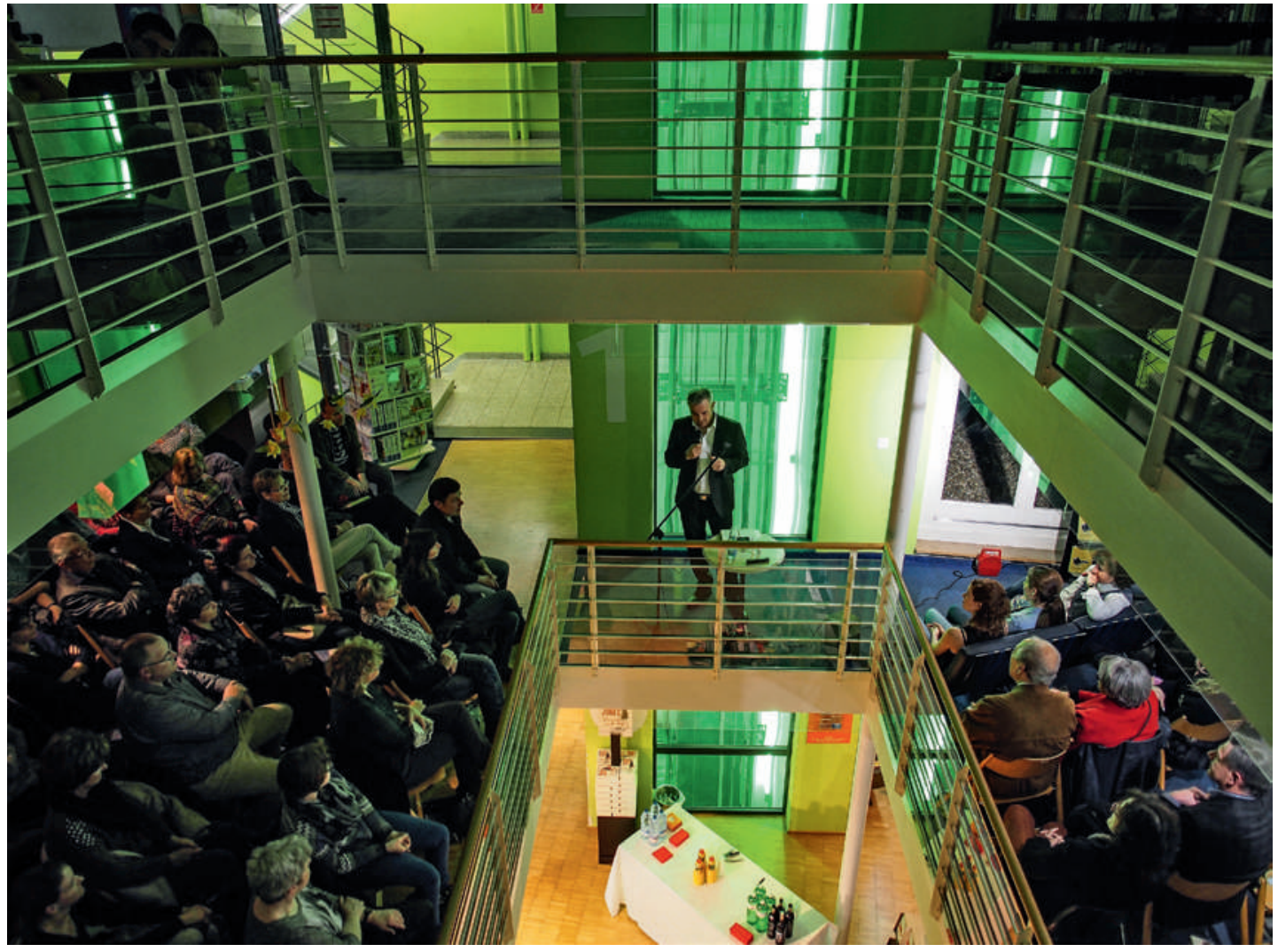

Alle lauschen Peter Hänni. Er stellt seinen neusten Krimi vor, in dem aus Harmonie schnell kriminelle Energie wird.

Er sei «einer der erfolgreichen Autoren der Region», sagt die Buchhändlerin, die zur Vernissage begrüsst. Spezialität: Krimis. Tatsächlich scheint tout Solothurn gekommen zu sein. Darunter wohl viele Patientinnen und Patienten des Autors, denn dieser ist ja vor allem einer der erfolgreichen Ärzte der Region. Hals, Nasen und Ohren sind in seinem Hauptberuf die Spezialitäten. «Hasenohren» habe mal einer verstanden, erzählt er dem amüsierten Publikum, und dieses erhält damit gleich den Beweis für die Aussage der Buchhändlerin, Peter Hänni sei auch ein erfolgreicher Entertainer mit einer humoristischen Ader.

In seinem jüngsten Roman, den er in der Buchhandlung Lüthy vorstellt, gibt es offenbar weniger zu lachen: Schnell wird da ein harmonisches Miteinander zum kriminellen Ereignis, das wird schon in den Passagen klar, die Hänni vorliest.
Es gibt im Schweizer Mittelland mehrere Ärzte, die Krimis schreiben. Warum? Ist ihr Alltag nicht schon blutig genug? Oder vielleicht gar zu wenig spannend? Anamnese und Diagnose eines Arztes seien ähnliche Aufgaben wie diejenigen eines Kommissars, wenn dieser einen schwierigen Fall aufzuklären habe, hat mal einer gesagt. «Ja», sagt Peter Hänni, «beide müssen gut beobachten können. Vor allem aber: Als Autor und als Arzt muss man Sitzleder haben, Ausdauer und Durchhaltewillen, das ist das Verbindende». Er sei zum Schreiben über seine persönlichen Vorlieben als Leser gekommen. In aller Regel habe er zu wenig Zeit und zu wenig Lust für anspruchsvolle Literatur. «Schon im Gymer habe ich mich durch die Literaturliste gekämpft.»

Er lasse sich von Büchern gerne unterhalten, wolle relaxen beim Lesen. Als Autor habe er keine weitergehenden Ansprüche. «Fun, Abwechslung, 
etwas Spannung und ein paar ruhige Stunden - das schätze ich, und das schätzen auch meine Leserinnen und Leser.»

Und wann findet einer, der sonst schon wenig Zeit hat, Zeit zum Schreiben? «Auch Ärzte haben ihre Hobbies», sagt Hänni, gedanklich schreibe er beispielsweise beim Autofahren oder auf dem Skilift, richtig dann an einem Samstagnachmittag oder in

\section{«Auch Ärzte haben ihre Hobbies.»}

den Ferien. «Ich bin ein Quartalsschreiber. Deshalb habe ich auch nicht den gleichen Druck wie einer, der vom Schreiben lebt.»

Thematisch sei der Alltag eines Arztes natürlich schon ein reicher Fundus für seine Nebentätigkeit als Krimi-Autor. Wobei nicht bloss eine Psychiaterin wie Esther Pauchard ein Gespür für die Abgründe des menschlichen Daseins haben könne, sondern beispielsweise auch ein Allgemeinpraktiker wie Paul Wittwer, sagt Hänni. Oder ein Oto-Rhino-Laryngologe wie Peter Hänni, sagt sein Erfolg.

Bei «Rosas Blut» (2008), «Samenspende» (2009) und «Freitod, der 13.» (2011) war die direkte inhaltliche Verbindung zur Medizin klar, es ging unter anderem um Leihmütter und Sterbebegleitung. «Von diesem direkten Bezug zu meinem Hauptberuf wollte ich diesmal etwas wegkommen», sagt Hänni, und er geht in seinem vierten Kriminalroman auch geografisch etwas weiter weg. Nicht Bern und Solothurn sind diesmal seine Schauplätze, sondern Städte und Landschaften in der Kap-Region in Südafrika. Präzise Beschreibungen machen sofort klar: Hänni war selber dort. Wie seine Protagonisten auch mit einer Harley. Und mit Kollegen, die etwa alle zwei Jahre eine gemeinsame Töffreise unternehmen. Eben erst fuhren sie durch Osteuropa. «Die Südafrika-Reise haben wir 2011 zu fünft gemacht, und schon damals war klar, dass ich sie zum Kriminalroman aufpeppen würde. Wirklich passiert ist das, was als banales Ereignis jetzt auch der Kern meines Krimis ist, nämlich dass einer seinen Töffschlüssel verloren hat.» Und gleich wie im Roman sei eine solche Reise auch in der Wirklichkeit eine willkommene Abwechslung zum Alltag. Das manifestiere sich unter anderem in der Sprache: Auf einer Töfftour mit Kumpels sei diese schon einiges derber als in einer Arztpraxis, wo man immer freundlich und differenziert zu sein habe, sagt Peter Hänni dazu.

Der grosse Unterschied zwischen Realität und Fiktion: Bei «Boarding Time» kommen von den fünf Freizeit-Bikern, die gemeinsam zum Abenteuer gestartet sind, nur noch drei lebend in die Schweiz zurück. Und diese sind heillos zerstritten. «Wir waren nach unserer Reise alle gesund und gut gelaunt», erzählt Hänni, «und wir haben uns heute noch gern.»

Ob das allerdings nach der Veröffentlichung des

\section{«Ich bin ein Quartalsschreiber.»}

Romans so bleibe, müsse sich erst weisen, fügt er an der Vernissage scherzhaft hinzu. Die richtigen Töffkumpels tauchen dort, offiziell zumindest, nicht auf. Auf den Pendants, die im Roman Orthopäde, Investmentbanker oder Hotelier sind, lastet der Verdacht, eine schwarze Hotelangestellte vergewaltigt und ermordet zu haben.

Aber im richtigen Leben ist ja zum Glück alles anders.

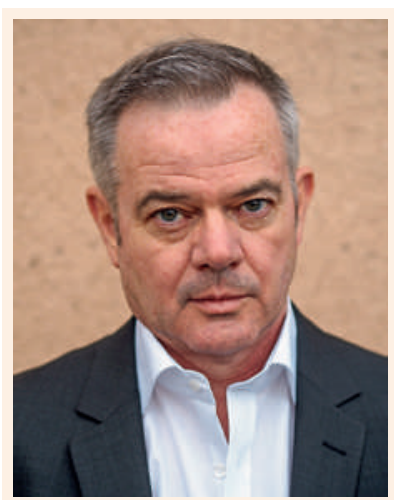

Peter Hänni wurde 1958 in Bern geboren. Er war Kellner, Metzgergehilfe, Bauarbeiter und Taxifahrer, bevor er an der Uni Bern einige Semester Jus und schliesslich Medizin studierte. Heute ist er Facharzt für Hals-, Nasen-, Ohrenkrankheiten und für Hals- und Gesichtschirurgie. Seit 1996 führt er in Solothurn eine eigene Privatpraxis. «Rosas Blut», sein erster Kriminalroman, erschien 2008 beim Schweizerischer Ärzteverlag EMH. Peter Hänni lebt mit seiner Familie in Lommiswil bei Solothurn. 